\title{
Computational modelling prediction and clinical validation of impact of benralizumab on airway smooth muscle mass in asthma
}

To the Editor:

Asthma is a heterogeneous condition characterised by chronic inflammation and airway remodelling. T2-mediated airway inflammation, characterised by interleukin (IL)-4, IL-5 and IL-13, has been the recent focus of pharmacotherapy, with a host of targeted biologic agents now available to clinicians [1]. The anti-IL-5 receptor agent, benralizumab, causes depletion of eosinophils and basophils via antibodydependent cell-mediated cytotoxicity in blood; however, the mechanism of action in the tissue is uncertain. Benralizumab has reduced exacerbations and improved lung function in clinical trials.

Airway remodelling describes structural changes including goblet cell hyperplasia, subepithelial matrix deposition, neoangiogenesis of bronchial vasculature and increased airway smooth muscle (ASM) mass, which can result from both cellular hypertrophy and hyperplasia [2]. ASM mass predicts airflow limitation, and is associated with reduced luminal area on computed tomography scan [3]. We showed recently that there was reduction in ASM mass in a prospective, randomised, placebo-controlled trial of the prostaglandin D2 antagonist fevipiprant, through reduced myofibroblast recruitment in concert with attenuation of eosinophilic inflammation [4]. A novel approach, integrating an agent-based computational model [5], clinical trial results and in vitro data was employed to provide insight into these mechanisms. The computational model was based on airway cell interactions, with perturbations in the interactions in order to recapitulate the changes observed following epithelial injury in health and disease. This approach allows us to simulate the airway remodelling in severe asthma, providing a "virtual patient" model.

We hypothesised that this computational model could be applied to predict the impact of benralizumab on the ASM bundle in asthma and that benralizumab reduces ASM mass. To test this hypothesis, we aimed to evaluate the effect of benralizumab upon ASM mass through analysis of bronchial biopsies obtained from previous clinical trials and to validate the computational model.

Bronchial biopsies were obtained from subjects with eosinophilic asthma participating in a previous phase 1 multicentre randomised double-blind placebo-controlled trial to determine the safety and tolerability of benralizumab [6]. In the trial, patients were randomised to a single intravenous dose, or three monthly subcutaneous doses, of benralizumab or placebo.

Formalin-fixed, paraffin-embedded 4 - $\mu \mathrm{m}$ sections were evaluated from 15 subjects receiving benralizumab and 10 subjects receiving placebo, pre- and post-treatment. Four subjects had biopsies available from two follow-up visits; the time average change was derived for these subjects $(n=54)$. Immunostaining for $\alpha$-smooth muscle actin was performed using Autostainer Link 48 (Agilent, Santa Clara, CA, USA) with appropriate isotype controls. Morphological analysis was performed on scanned sections using the Axio Scanner and AxioCam HRc digital camera (Carl Zeiss, Jena, Germany). ZEN 2012V 2.3 desk software was used to perform image analysis on total ASM, epithelium and lamina propria areas. The percentage of ASM mass was presented as the mean of at least two replicates from two different sites. All morphometry measurements and counting were performed by one observer, blinded to participant identification, treatment allocation and study visit. Comparisons were then made within-group before and after treatment allocation and between interventions. Numbers of eosinophils in the lamina propria pre- and

@ERSpublications

Benralizumab leads to a reduction in ASM mass in asthmatic airways, which can be predicted using a computational model http://bit.ly/2OwNMBy

Cite this article as: Chachi L, Diver S, Kaul H, et al. Computational modelling prediction and clinical validation of impact of benralizumab on airway smooth muscle mass in asthma. Eur Respir J 2019; 54: 1900930 [https://doi.org/10.1183/13993003.00930-2019]. 

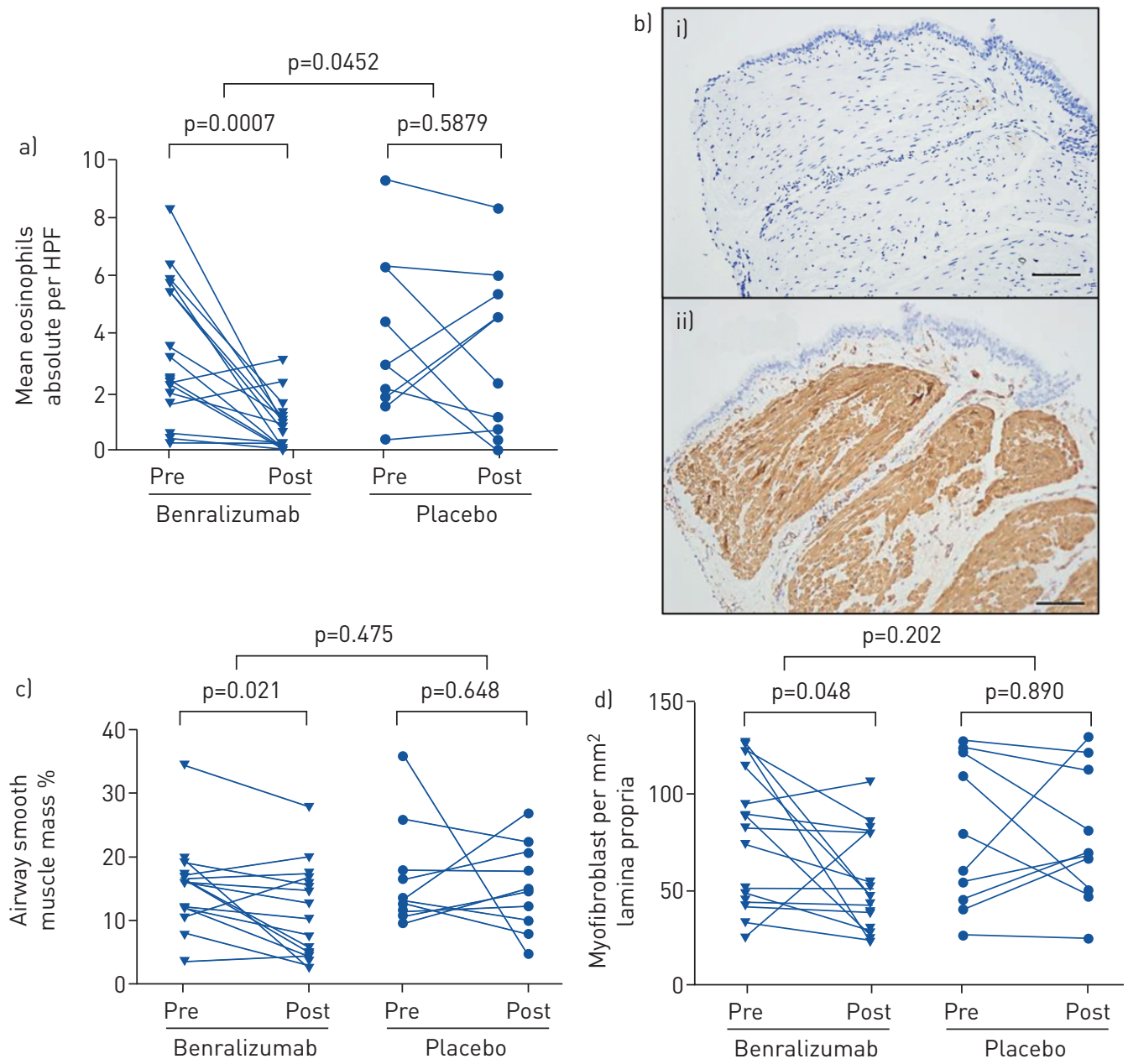

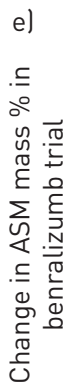

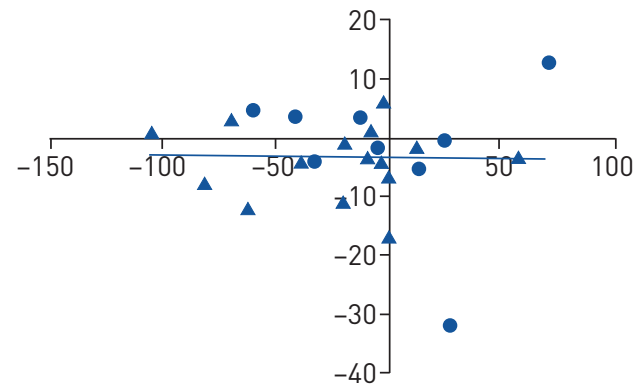

\section{A Benralizumab}

- Placebo

Change in myofibroblast number per $\mathrm{mm}^{2}$ lamina propria in benralizumab trial

FIGURE 1 Airway smooth muscle (ASM) mass in asthma is reduced by benralizumab. a) Airway eosinophils labsolute per high-power field (HPF)] for individual subjects used in the benralizumab study. b) Representative photomicrograph of a bronchial biopsy from a subject with asthma in the benralizumab trial, showing increased ASM: i) isotype control (IC) and ii) brown-stained $\alpha$-smooth muscle actin (SMA); scale bars $=100 \mu \mathrm{m}$. c) ASM mass, measured by percentage $\alpha$-SMA-positive area, in bronchial biopsies from asthmatic subjects before treatment (Pre) and after treatment (Post) with benralizumab ( $n=15$ ) or placebo $(n=10)$. A two-tailed paired t-test was used for within-group comparisons ( $p=0.021$ and $p=0.648)$, and a two-tailed unpaired t-test was used to compare the difference in ASM mass observed after treatment with benralizumab to that seen in the placebo group ( $p=0.475)$. d) Myofibroblast count in lamina propria as measured positive stained cells in $\mathrm{mm}^{2}$ in bronchial biopsies from asthmatic subjects before treatment (Pre) and after treatment (Post) with benralizumab $(n=15)$ or placebo $(n=10)$. A two-tailed paired t-test was used for within group comparisons ( $p=0.045$ and $p=0.890)$, and a two-tailed unpaired t-test was used to compare the difference in ASM mass observed after treatment with benralizumab to that seen in the placebo group $(p=0.202)$. e) Correlation between change in myofibroblast number in the lamina propria and absolute change in ASM mass as a percentage of the total biopsy area (benralizumab $n=15$, placebo $n=10$; Spearman $r(95 \%$ Cl) $-0.09(-0.48-0.33) ; p=0.663)$. 
post-treatment were obtained from the original trial. GraphPad Prism (version 7.0; GraphPad, San Diego, CA, USA) was used for statistical analysis. $\mathrm{p}<0.05$ was considered statistically significant.

The number of eosinophils in the lamina propria decreased significantly following treatment with benralizumab (66.4\% reduction) and compared with the placebo group (88\% post-treatment reduction in eosinophils relative to placebo) (figure 1a). Based on mechanism of action, in parallel with the observed mean change in eosinophil number in the lamina propria the model predicted that benralizumab would have had a pro-apoptosis efficiency of $47 \%$, with a corresponding consequent $29 \%$ relative reduction in ASM mass.

A representative photomicrograph of a bronchial biopsy from an asthmatic subject at baseline, demonstrating an increase in ASM mass is shown in figure 1b. The absolute percentage ASM mass (mean \pm SEM) decreased significantly after treatment with benralizumab $(-4.1 \pm 1.6 \%, \mathrm{p}=0.021 ; \mathrm{n}=15)$, while there was no significant change in those who received placebo $(-1.8 \pm 3.7 \%, \mathrm{p}=0.648 ; \mathrm{n}=10)$. The mean difference $(95 \% \mathrm{CI})$ between groups was not significant $(-2.6 \%,-10.0-4.8 \% ; \mathrm{p}=0.475)$ (figure $1 \mathrm{c}$ ). The mean relative percentage reduction in ASM mass following treatment with benralizumab versus placebo was $29 \%$, consistent with the prediction generated by our computational model.

Additionally, in the benralizumab group, there was a reduction in the number of tissue myofibroblasts $(-23.4 \pm 10.8, \mathrm{p}=0.048 ; \mathrm{n}=15)$, with no significant change observed in the control group $(-1.7 \pm 12.1$, $\mathrm{p}=0.890 ; \mathrm{n}=10)$. The mean difference $(95 \% \mathrm{CI})$ between groups was not significant $(-21.7,-55.8-12.4$; $\mathrm{p}=0.202$ ) (figure 1d). No correlation was observed between the change in number of myofibroblasts in the lamina propria and the change in ASM mass as a percentage of the total biopsy area (benralizumab $\mathrm{n}=15$, placebo $\mathrm{n}=10$, Spearman $\mathrm{r}, 95 \% \mathrm{CI}-0.09,-0.48-0.33$; $\mathrm{p}=0.663$ ) (figure 1e).

ASM cells do not express IL-5R, and therefore the effects of benralizumab upon ASM mass are likely to be an indirect effect of reducing eosinophilic inflammation. The reduction in eosinophils in the bronchial biopsies following benralizumab was substantial, but perhaps less than might be predicted by the mechanism of action, possibly due to additional factors promoting eosinophil survival in tissue. Transcription growth factor (TGF)- $\beta$ has been implicated in airway remodelling as a potent controller of fibroblast/myofibroblast function through production of extracellular matrix proteins [7] and has been highlighted as a key effector in promoting ASM proliferation and migration [8]. Eosinophils constitute the majority of TGF- $\beta$-expressing cells in asthmatic lungs [9]. Therefore, the reduction in ASM observed in response to benralizumab may relate to reduced levels of airway TGF- $\beta$ or other important growth factors or chemokines. This possibility warrants further investigation in future trials.

One limitation of this study is its sample size. Although we have studied the only available bronchial biopsy material obtained in a placebo-controlled trial of benralizumab, the number of subjects is small, and thus our failure to show a difference between benralizumab and placebo upon ASM mass might be due to underpowering. Future trials will need to consider larger numbers of subjects that undergo multiple biopsies. The study duration is short and further effects on airway remodelling might have been observed with a longer trial period. Notwithstanding these limitations, the similar results from the computational model and the clinical trial are remarkable and do validate the utility of the model in predicting the impact of interventions upon airway remodelling in a "virtual patient".

In summary, we demonstrate that benralizumab leads to a reduction in ASM mass in asthma, which can be predicted using a computational modelling approach.

Latifa Chachi $\circledast^{1,4}$, Sarah Diver ${ }^{1,4}$, Himanshu Kaul ${ }^{2,4}$, Marlon C. Rebelatto ${ }^{3}$, Anmarie Boutrin ${ }^{3}$, Patricia Nisa ${ }^{1}$, Paul Newbold ${ }^{3}$ and Christopher Brightling ${ }^{1}$

${ }^{1}$ IHR Leicester Biomedical Research Centre, Respiratory, Dept of Respiratory Sciences, Glenfield Hospital, University of Leicester, Leicester, UK. ${ }^{2}$ University of British Columbia, Vancouver, BC, Canada. ${ }^{3}$ MedImmune, AstraZeneca, Gaithersburg, MD, USA. ${ }^{4}$ These authors contributed equally.

Correspondence: Christopher E. Brightling, Institute for Lung Health, NIHR BRC Respiratory Medicine, University of Leicester, Respiratory Sciences, Groby Road, Leicester, UK. E-mail: ceb17@leicester.ac.uk

Received: 9 May 2019 | Accepted after revision: 25 July 2019

This study is a post-analysis from a previous clinical trial (NCT00659659).

Conflict of interest: L. Chachi has nothing to disclose. S. Diver has nothing to disclose. H. Kaul reports grants from AirPROM, during the conduct of the study. M.C. Rebelatto is an employee of and owns shares in AstraZeneca. A. Boutrin is an employee of and holds stock options in AstraZeneca. P. Nisa has nothing to disclose. P. Newbold is an employee of and owns shares in AstraZeneca. C. Brightling reports grants and personal fees for consultancy from GlaxoSmithKline, AstraZeneca/Medimmune, Novartis, Chiesi, Roche/Genentech and Boehringer Inglheim, personal fees for consultancy from Vectura, Theravance, PreP, Gilead, Sanofi/Regeneron, Teva, Gossamer and 4DPharma, grants from Pfizer and Mologic, outside the submitted work. 


\section{References}

1 Diver S, Russell RJ, Brightling CE. New and emerging drug treatments for severe asthma. Clin Exp Allergy 2018; 48: 241-252.

2 Pascual RM, Peters SP. Airway remodeling contributes to the progressive loss of lung function in asthma: an overview. J Allergy Clin Immunol 2005; 116: 477-486.

3 Hartley RA, Barker BL, Newby C, et al. Relationship between lung function and quantitative computed tomographic parameters of airway remodeling, air trapping, and emphysema in patients with asthma and chronic obstructive pulmonary disease: a single-center study. J Allergy Clin Immunol 2016; 137: 1413-1422.

4 Saunders $\mathrm{R}$, Kaul $\mathrm{H}$, Berair $\mathrm{R}$, et al. $\mathrm{DP}_{2}$ antagonism reduces airway smooth muscle mass in asthma by decreasing eosinophilia and myofibroblast recruitment. Sci Transl Med 2019; 11: eaao6451.

5 Kaul H, Ventikos Y. Investigating biocomplexity through the agent-based paradigm. Brief Bioinform 2015; 16: $137-152$.

6 Laviolette M, Gossage DL, Gauvreau G, et al. Effects of benralizumab on airway eosinophils in asthmatic patients with sputum eosinophilia. J Allergy Clin Immunol 2013; 132: 1086-1096.

7 Minshall EM, Leung DY, Martin RJ, et al. Eosinophil-associated TGF- $\beta_{1}$ mRNA expression and airways fibrosis in bronchial asthma. Am J Respir Cell Mol Biol 1997; 17: 326-333.

8 Brightling CE, Gupta S, Gonem S, et al. Lung damage and airway remodelling in severe asthma. Clin Exp Allergy 2012; 42: 638-649.

9 Flood-Page P, Menzies-Gow A, Phipps S, et al. Anti-IL-5 treatment reduces deposition of ECM proteins in the bronchial subepithelial basement membrane of mild atopic asthmatics. J Clin Invest 2003; 112: 1029-1036.

Copyright @eERS 2019 